\title{
Induction of apoptosis and stress response in ovarian carcinoma cell lines treated with ST1926, an atypical retinoid
}

\author{
V Zuco ${ }^{1}$, C Zanchi ${ }^{1}$, G Cassinelli ${ }^{1}$, C Lanzi ${ }^{1}$, R Supino ${ }^{1}$, \\ C Pisano ${ }^{2}$, R Zanier ${ }^{2}$, V Giordano ${ }^{2}$, E Garattini ${ }^{3}$ and F Zunino ${ }^{*}, 1$ \\ 1 Istituto Nazionale Tumori, Via Venezian 1, 20133 Milan, Italy; \\ 2 Sigma-Tau, 00040 Pomezia, Rome, Italy; \\ ${ }^{3}$ Istituto Ricerche Farmacologiche Mario Negri, 20157 Milan, Italy \\ * Corresponding author: F Zunino. Tel: + 39-02-23902267; \\ Fax: + 39-02-23902692; E-mail: franco.zunino@ istitutotumori.mi.it
}

Received 17.6.03; revised 19.6.03; accepted 19.6.03; published online 5.12.03 Edited by L. Fesus

\begin{abstract}
To understand the molecular mechanisms mediating apoptosis induction by a novel atypical retinoid, ST1926, the cellular response to drug treatment was investigated in IGROV-1 ovarian carcinoma cells carrying wild-type p53 and a cisplatin-resistant p53 mutant subline (IGROV-1/Pt1). Despite a similar extent of drug-induced DNA strand breaks, the level of apoptosis was substantially higher in p53 wild-type cells. p53 activation and early upregulation of p53-target genes were consistent with p53-dependent apoptosis in IGROV-1 cells. Stress-activated protein kinases were activated in both cell lines in response to ST1926. This event and activation of AP-1 were more pronounced in IGROV-1/Pt1 cells, in which the modulation of DNA repair-associated genes suggests an increased ability to repair DNA damage. Inhibition of JNK or p38 stimulated ST1926-induced apoptosis only in IGROV-1 cells, whereas inhibition of ERKs enhanced apoptosis in both the cell lines. Such a pattern of cellular response and modulation of genes implicated in DNA damage response supports that the genotoxic stress is a critical event mediating drug-induced apoptosis. The results are consistent with apoptosis induction through p53-dependent and independent pathways, regulated by MAP kinases, which likely play a protective role.

Cell Death and Differentiation (2004) 11, 280-289. doi:10.1038/ sj.cdd. 4401304

Published online 5 December 2003
\end{abstract}

Keywords: atypical retinoids; apoptosis; DNA damage; stress response

\footnotetext{
Abbreviations: RAR, retinoic acid receptor; JNK, c-Jun Nterminal kinase; AP-1, activator protein-1; RPA, replicationprotein A; PCNA, proliferating cell nuclear antigen; ERK, extracellular signal-regulated kinase; MAPK, mitogen-activated protein kinase; TUNEL, TdT-uridine nucleotide end-labelling technique; PI, propidium iodide; SAPK, stress-activated protein kinase; MEK, MAPK/ERK kinase; ATF, activation transcription factor; NF- $\kappa \mathrm{B}$, nuclear factor kappaB; ROS, reactive oxygen
}

species; DMSO, dimethyl sulfoxide; SDS-PAGE, sodium dodecyl sulfate-polyacrylamide gel electrophoresis; PMSF, phenylmethylsulfonyl fluoride

\section{Introduction}

Atypical retinoids represent a novel class of potentially useful agents for cancer treatment. Classical retinoids are known to modulate cell growth and differentiation through their binding to retinoid nuclear receptors. In contrast, atypical retinoids, including CD437 and analogs, exhibit a unique mechanism of action, resulting in growth-inhibitory and proapoptotic activity that is not receptor-mediated. ${ }^{1}$ In particular, CD437 is referred to as RAR- $\gamma$-selective retinoid, but the role of activation of RAR receptor in the CD437 induction of apoptosis is still controversial. Indeed, atypical retinoids exert growth-inhibitory activity and induce apoptosis in retinoid-resistant cells, and these cellular effects cannot be blocked by RARantagonists. $^{2-4}$ Multiple mechanisms, including activation of JNK and p38, have been implicated in apoptosis induced by atypical retinoids. ${ }^{5}$ However, the mechanism of the growthinhibitory activity and the cellular basis of the broad spectrum of activity of these compounds remain largely unknown. CD437 increases the level of p53 protein, which plays a critical role in DNA damage response, suggesting the involvement of a genotoxic stress in the mechanism of antiproliferative and proapoptotic activity. ${ }^{6}$ Moreover, human tumor cell lines carrying wild-type p53 have been reported to be more susceptible to CD437-induced apoptosis, ${ }^{6}$ and this cellular response is reminiscent of effects elicited by DNA-damaging agents. Again, activation of the JNK pathway described for atypical retinoids ${ }^{5}$ is another typical aspect of cellular response to genotoxic stress. ${ }^{7,8}$

Recently, we have reported that a related compound, ST1926 (Figure 1), characterized by an almost complete loss of ability to activate retinoid receptors, exhibited a high antiproliferative potency and marked ability to induce apoptosis. ${ }^{9}$ In addition, ST1926 was found to induce DNA damage under conditions causing apoptotic effects. In an attempt to better elucidate the molecular and cellular basis of the apoptosis induced by ST1926, we have performed a study in an ovarian carcinoma cell system, including the parental IGROV-1 cells carrying wild-type p53 and a subline, IGROV1/Pt1, selected for resistance to cisplatin and characterized by p53 mutation. The study was designed to investigate the relevant aspects of cellular response to ST1926 in different biological contexts, with particular reference to the significance of stress-activated protein kinase pathways after druginduced cellular injury. We found that the cell subline with mutant p53 was less sensitive to ST1926-induced apoptosis, and exhibited an enhanced activation of stress-activated kinases. Our results indicate a protective function of the MAP 


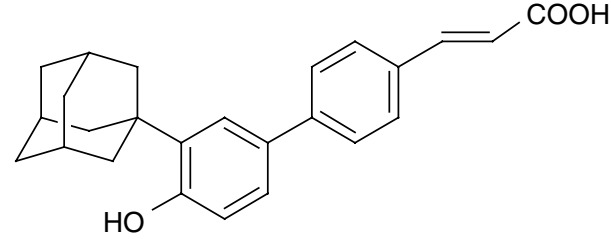

Figure 1 Chemical structure of ST1926

kinase family members in ovarian carcinoma cells treated with ST1926, and are consistent with activation of a DNA damage response.

\section{Results}

\section{Antiproliferative effects and apoptosis induction}

The antiproliferative activity of ST1926 against the two ovarian carcinoma cell lines was determined after $72 \mathrm{~h}$ of drug exposure. The $\mathrm{IC}_{50}$ values were $0.23 \pm 0.09$ and $0.43 \pm 0.08 \mu \mathrm{M}$ for IGROV-1 and IGROV-1/Pt1 cells, respectively. The $\mathrm{IC}_{50}$ or $\mathrm{IC}_{80}$ concentrations in further experiments are referred to values determined under these conditions (i.e. $72 \mathrm{~h}$ exposure). Further cellular/biochemical pharmacology studies were performed at equitoxic drug levels to allow a comparison of the cellular response under conditions causing the same antiproliferative effects.

The apoptotic response of IGROV-1 and IGROV-1/Pt1 cells to ST1926 was determined by TUNEL assay and PI staining. After $24 \mathrm{~h}$ of exposure to drug concentrations corresponding to the respective $\mathrm{IC}_{80}(0.3 \mu \mathrm{M}$ for IGROV-1 and $0.6 \mu \mathrm{M}$ for IGROV-1/Pt1 in the $72 \mathrm{~h}$ antiproliferative test), DNA fragmentation typical of apoptosis was detected in $30 \%$ of IGROV-1 cells, and only in $10 \%$ of IGROV-1/Pt1 cells (Figure 2 ). The extent of apoptotic cells found after $72 \mathrm{~h}$ exposure (65 and $30 \%$ in IGROV-1 and IGROV-Pt1 cells, respectively), was consistent with a reduced sensitivity of the cisplatin-resistant subline to ST1926. The results were supported by the fluorescence miscroscopy observation of PI-stained cells (not shown).

\section{Caspase activation}

The activation of caspases associated with cleavage of substrates such as PARP is a hallmark of apoptosis. ${ }^{10}$ We examined the effect of ST1926 on caspases and PARP cleavage by Western blot analysis after different times of treatment (Figure 3). In IGROV-1 cells, exposure to ST1926 resulted in activation of caspases 3,8 and 9, already detectable after $24 \mathrm{~h}$ of treatment. The activation of caspase 3 was confirmed by the appearance of the $85 \mathrm{kDa}$ PARP cleavage product. Consistent with the reduced apoptotic response to ST1926 in IGROV-1/Pt1 cells, there was a weak activation of caspases 3 and 9, associated with delayed PARP fragmentation. No activation of caspase 8 was detectable in the latter cell line.

\section{Modulation of apoptosis-related proteins}

To investigate the pathways involved in the induction of apoptosis by ST1926 in the two ovarian carcinoma cell lines, the expression of apoptosis-related proteins was analyzed overtime (from 6 to $72 \mathrm{~h}$ ) by Western blotting. Figure 4a shows that drug treatment $\left(\mathrm{IC}_{80}\right)$ caused an early increase in p53 protein levels in IGROV-1 cells expressing a wild-type p53. On the contrary in IGROV-1/Pt1 cells, which express a mutated $\mathrm{p} 53$, no modulation of $\mathrm{p} 53$ was observed. The $\mathrm{p} 21^{\mathrm{WAF} 1}$ protein was upregulated in both cell lines by $6 \mathrm{~h}$ after drug treatment. This modulation was not surprising in IGROV-1/Pt1, since regulation of $\mathrm{p} 21^{\mathrm{WAF} 1}$ expression may be mediated by a p53independent mechanism. ${ }^{11}$ An increase of the proapoptotic Bax protein was found in IGROV-1 cells within $24 \mathrm{~h}$ of treatment, whereas no changes in the levels of the antiapoptotic Bcl-2 protein were found after treatment with ST1926 in both cell lines (Figure 4b).

\section{Cell cycle perturbation}

In order to examine the effects of ST1926 on cell cycle progression, IGROV-1 and IGROV-1/Pt1 cells were exposed to equitoxic concentrations of ST1926 $\left(\mathrm{IC}_{80}\right)$ for 24,48 and $72 \mathrm{~h}$ (Figure 5). The DNA content analysis by flow cytometry showed that IGROV-1 cells underwent a persistent G1 arrest after exposure to the drug. Consistent with the early apoptotic response of IGROV-1 cells, a marked sub-G1 peak was already found at $24 \mathrm{~h}$ and increased at $48-72 \mathrm{~h}$. A different pattern of cell cycle perturbation was evident in the $p 53$ mutant IGROV-1/Pt1 cell line, which exhibited an appreciable accumulation also in $S$ phase and a late appearance of the sub-G1 peak.

\section{DNA damage}

Since the pattern of cellular response to ST1926 involving p53 and drug-induced cell cycle arrest at $\mathrm{G} 1$ and $\mathrm{S}$ phases were reminiscent of the cellular response to DNA-damaging agents, ${ }^{12}$ we investigated the effects of the drug on DNA integrity by the alkaline elution technique. Following $6 \mathrm{~h}$ exposure to drug concentrations in a range between the $\mathrm{IC}_{50}$ and the $\mathrm{IC}_{90}$, the two cell lines exhibited a comparable extent of single-strand DNA breaks (Figure 6). The conditions used for the alkaline elution assay do not allow one to detect double -strand breaks. The precise nature of the DNA damage remains to be determined.

To provide additional support to the formation of genotoxic damage, we further investigated the effect of the drug on modulation of proteins involved in the cellular response to DNA damage. RPA-2, a DNA single-strand binding protein essential for DNA replication, recombination and repair, becomes rapidly phosphorylated after treatment with DNAdamaging agents. ${ }^{13}$ Thus, we examined RPA-2 phosphorylation as a marker of DNA damage response. A slower migrating band, evidenced by the shift in the electrophoretic mobility and corresponding to phosphorylated RPA-2 (Figure 7a), was clearly detected after $6 \mathrm{~h}$ of drug exposure in both cell lines. RPA-2 phosphorylation was more evident and persistent in 
IGROV-1
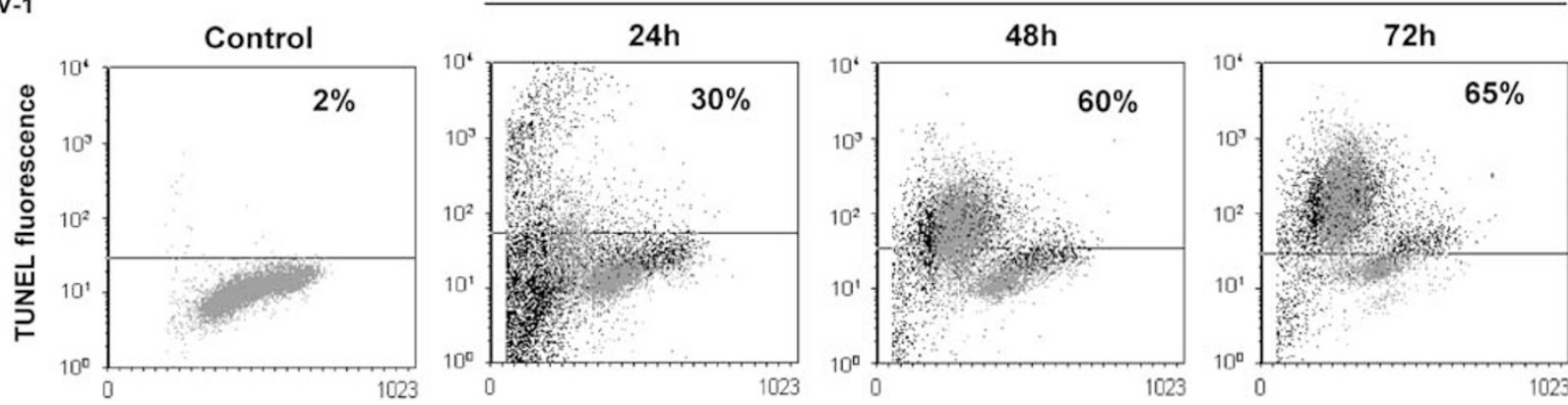

IGROV-1/Pt1
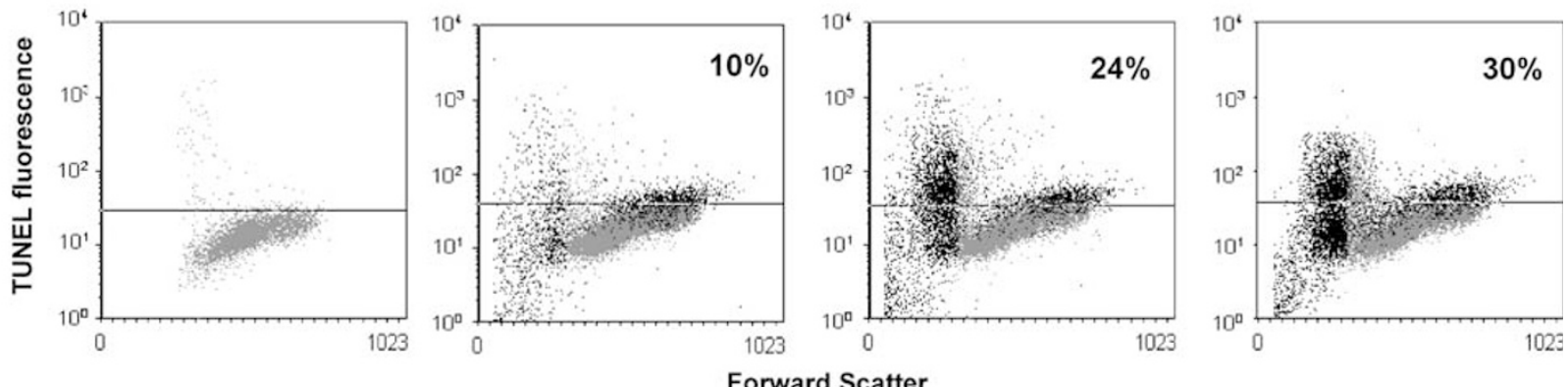

Forward Scatter

Figure 2 Time course of apoptosis in IGROV-1 and IGROV-1/Pt1 cells treated for the indicated times with 0.3 and $0.6 \mu \mathrm{M}$ ST1926, respectively, corresponding to the $I_{80}$. Apoptosis was determined by TUNEL assay and detected by FACS analysis. The plots are representative of three independent experiments. The percentages of TUNEL-positive cells are indicated in each panel

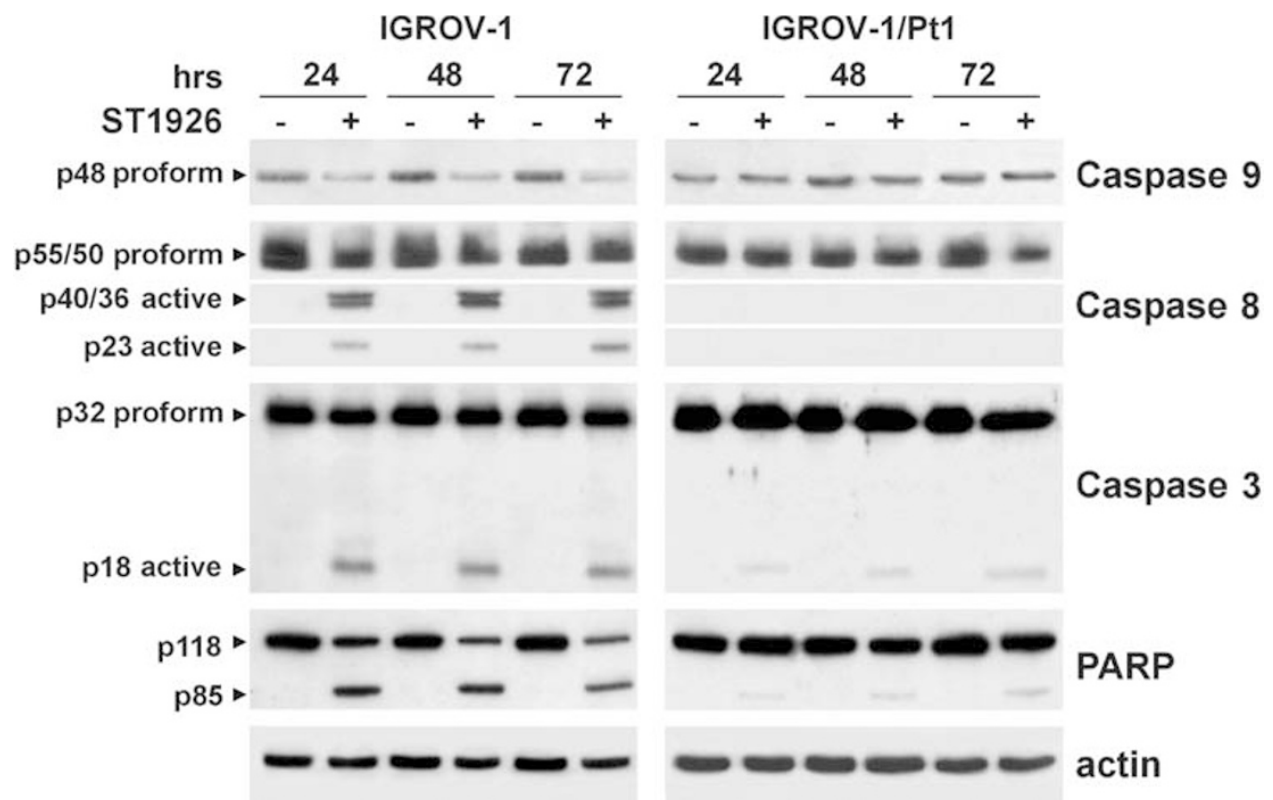

Figure 3 Cleavage of caspases and PARP in ovarian carcinoma cells exposed to ST1926 (+) or solvent $(-)$. Western blot analysis with specific antibodies was performed after 24,48 and $72 \mathrm{~h}$ exposure with 0.3 and $0.6 \mu \mathrm{M} \mathrm{ST1926}$ in IGROV-1 and IGROV-1/Pt1 cells, respectively $\left(\mathrm{IC}_{80}\right.$ at $\left.72 \mathrm{~h}\right)$. Actin is shown as control of the loading

IGROV-1/Pt1 cells, as clearly documented at longer times (up to $72 \mathrm{~h})$.

PCNA is a processivity factor for DNA polymerases, required for DNA synthesis and repair. Its recruitment to DNA repair sites involves the transition of the protein from a soluble to a chromatin-bound and detergent-insoluble state. ${ }^{14}$ Such behavior can be exploited to monitor the ongoing DNA repair. We determined the recruitment of soluble PCNA to insoluble sites by biparametric flow cytometric analysis, which enables to detect the cell cycle distribution of the insoluble 
a IGROV-1
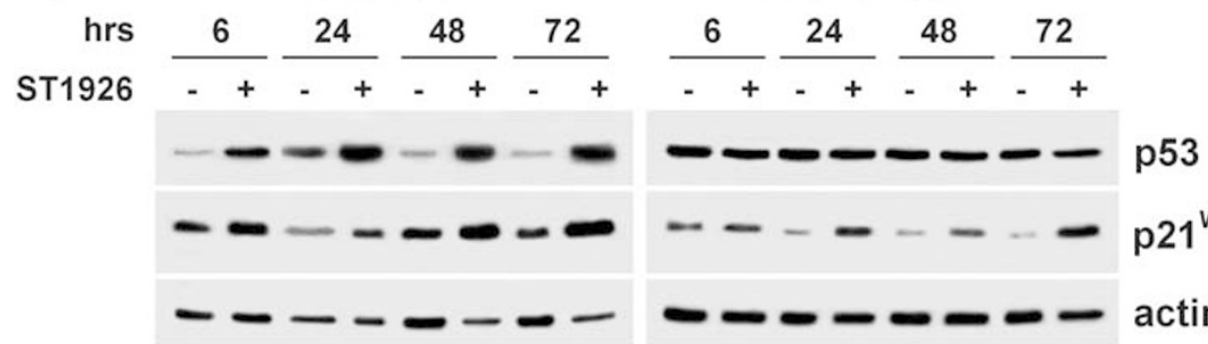

b

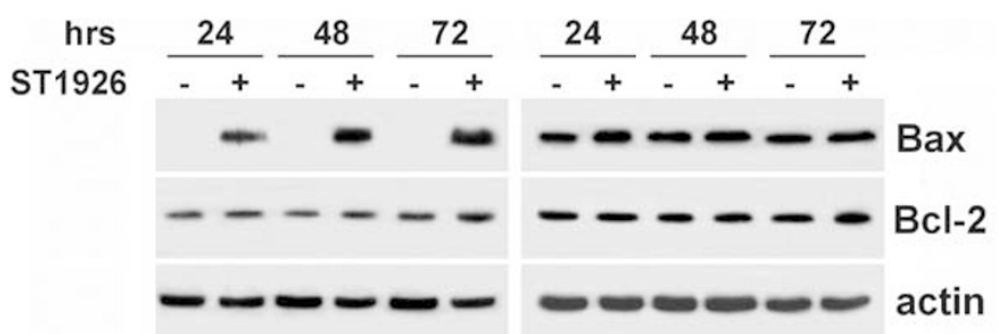

Figure 4 Expression of p53 and p21 WAF1 (a) or Bax and Bcl-2 (b) in IGROV-1 and IGROV-1/Pt1 cells treated with solvent $(-)$ or ST1926 at the respective IC 80 ( + ). Whole-cell extracts were prepared at the indicated times and analyzed by Western blotting. Actin is shown as control of protein loading

IGROV-1

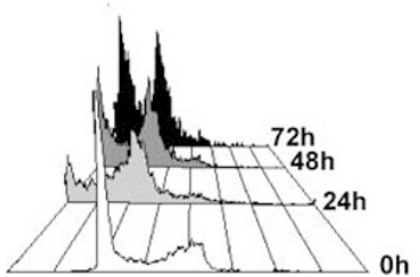

oh

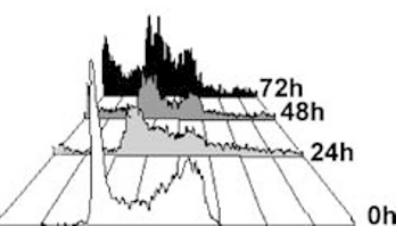

Figure 5 Cell cycle distribution of IGROV-1 and IGROV-1/Pt1 cells at different times after treatment with equitoxic concentrations of ST1926 $\left(\mathrm{IC}_{80}\right)$. A representative experiment is shown

form of the protein (Figure 7b). In both untreated cell lines, high levels of PCNA were present in S-phase cells, in which PCNA participates in DNA replication. The recruitment of PCNA to insoluble repair sites was detectable $6 \mathrm{~h}$ after treatment in G1 and G2/M phases only in IGROV-1/Pt1 cells. At longer times (24 and $48 \mathrm{~h}$ ), insoluble PCNA was not detectable in both the cell lines (not shown). Altogether, RPA2 phosphorylation and PCNA recruitment to repair sites support a cellular response to DNA damage induced by the drug in both cell lines. However, DNA repair-related processes could be detected for a more prolonged time in IGROV-1/Pt1 cells, thus suggesting that such response could contribute to the lower sensitivity of the subline to ST1926.

\section{Effect on ERKs and stress-activated MAP kinases}

Mitogen-activated protein kinase (MAPK) family members are activated by a variety of stimuli including cell injuries induced by DNA-damaging agents. ${ }^{15}$ We investigated the effect of ST1926 on MAPK pathways in the two ovarian carcinoma cell lines following treatment at the respective $\mathrm{IC}_{80}(0.3 \mu \mathrm{M}$ for IGROV-1 and $0.6 \mu \mathrm{M}$ for IGROV-1/Pt1 at $72 \mathrm{~h}$ ). Activation of

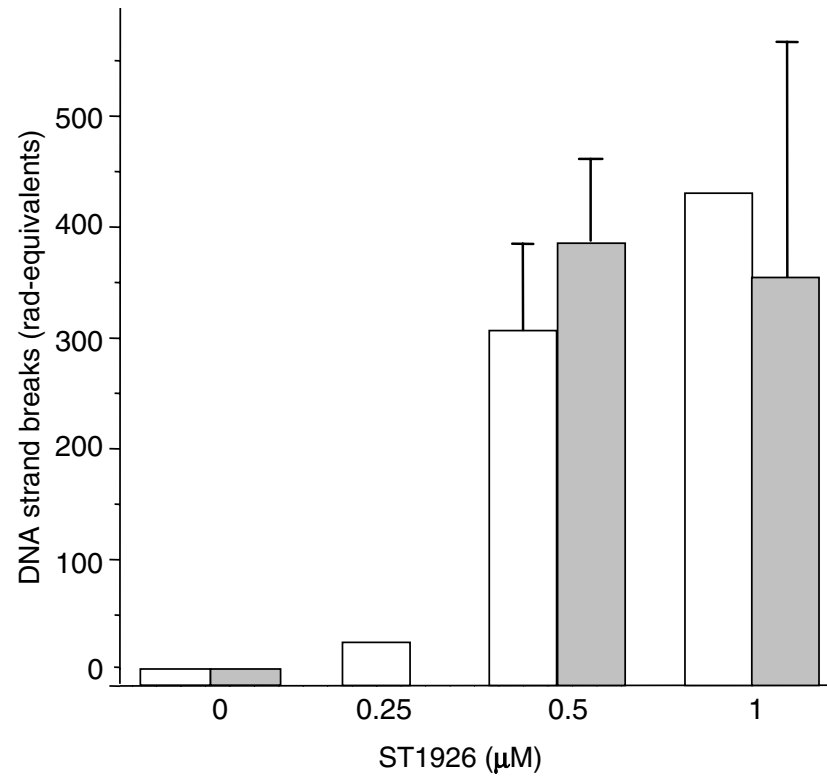

Figure 6 DNA single-strand breaks induced by ST1926. Cells were exposed to increasing drug concentrations for $6 \mathrm{~h}$, and then DNA breaks were measured by the alkaline elution assay. The results, expressed as rad-equivalents, are the mean \pm S.D. of three independent experiments. White columns: DNA breaks in IGROV-1 cells; grey columns: DNA breaks in IGROV-1/Pt1 cells

extracellular signal-regulated kinases (ERK1/2), detected in Western blots performed with phosphospecific antibodies, was not modulated by ST1926 during the first $6 \mathrm{~h}$ of treatment (Figure 8A), nor by more prolonged treatments (not shown). In contrast, ST1926 induced activation of the stress-activated kinases c-Jun- $\mathrm{NH}_{2}$-terminal kinases (JNKs) and p38. Although the phosphorylation of these kinases was enhanced by the drug in both the cell lines, the effect could be appreciated earlier in IGROV-1/Pt1 cells. In fact, activation 


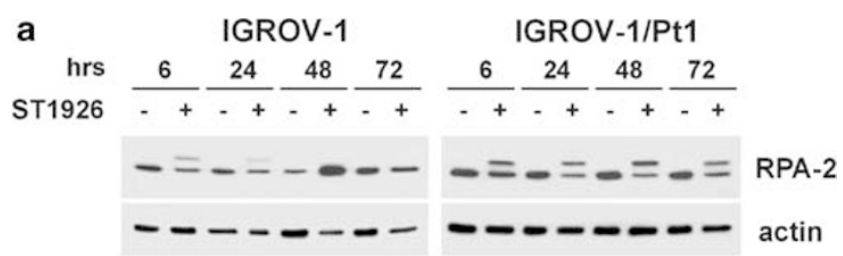

b

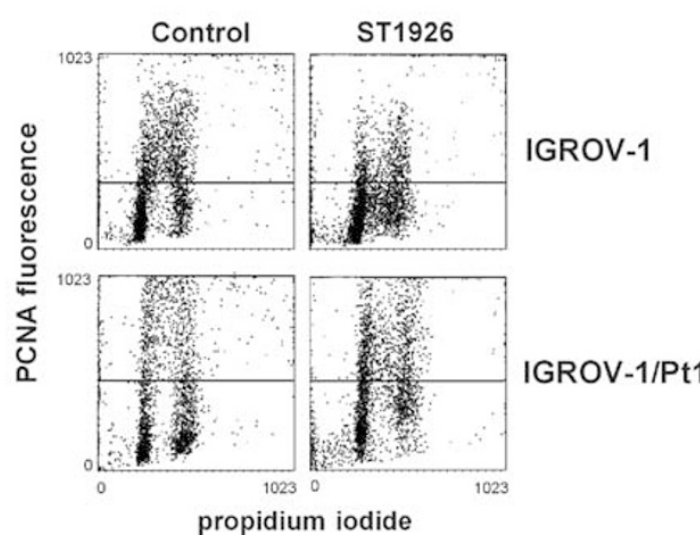

Figure 7 RPA-2 phosphorylation and PCNA distribution in IGROV-1 and IGROV-1/Pt1 cells exposed to equitoxic concentrations of ST1926 (IC 80$)$. (a) Western blot analysis of RPA-2 at different times of drug exposure. Actin level is shown as loading control. (b) Flow cytometric analysis of the cell cycle distribution of insoluble form of PCNA after $6 \mathrm{~h}$ of treatment. Biparametric dot plots of PCNA immunofluorescence versus DNA content (PI) are shown. One experiment representative of two independent experiments is reported

of JNKs and p38 was evident in IGROV-1/Pt1 cells following 1 and $3 \mathrm{~h}$ of treatment, respectively, whereas in IGROV-1 cells both kinases appeared weakly activated only after $6 \mathrm{~h}$ (Figure 8a). Again, dose-response experiments (Figure 8b) demonstrated that drug-induced activation of stress-activated MAP kinases, particularly p38, was more pronounced in IGROV-1/Pt1 cells compared to the parental cells.

\section{Effects of inhibitors of MAPK pathways on cell survival and apoptosis}

In order to examine in more detail the role of MAPKs in the response of the two ovarian carcinoma cell lines to ST1926, we used selective inhibitors of MAPK pathways in conjunction with ST1926 treatment. Compounds SP600125, PD169316 and PD98059 were used to inhibit cellular pathways depending on JNK, p38 and MEK-1, respectively. 5,16,17 The efficacy of the inhibitors on their own targets was checked biochemically, as described in Materials and Methods. Cell growth inhibition and apoptosis were determined after treatment with ST1926 combined with each inhibitor. The results reported in Figure 9 (top and middle panels) indicated that inhibition of JNK or p38 produced different effects in the two cell lines. In fact, in IGROV-1 cells treated with ST1926 (IC ${ }_{50}$ for $\left.72 \mathrm{~h}\right)$, the addition of SP600125 or PD169316 markedly reduced cell survival and increased the percentage of apoptosis. On the contrary, in IGROV-1/Pt1 cells treated with the $\mathrm{IC}_{50}$ for $72 \mathrm{~h}$ (not shown) or the $\mathrm{IC}_{80}$ for $72 \mathrm{~h}$ (Figure 9), cell growth inhibition and apoptosis were not affected by the presence of the two inhibitors. In combination experiments with PD98059, a

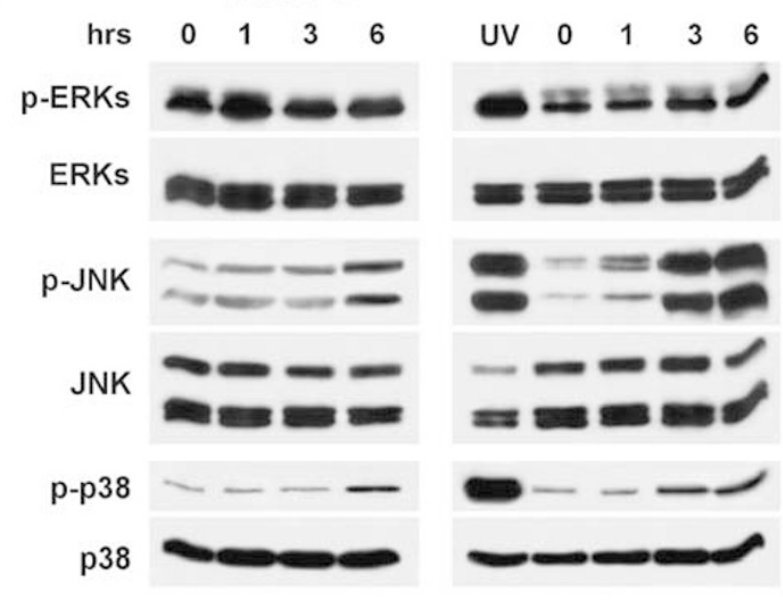

b IGROV-1 IGROV-1/Pt1

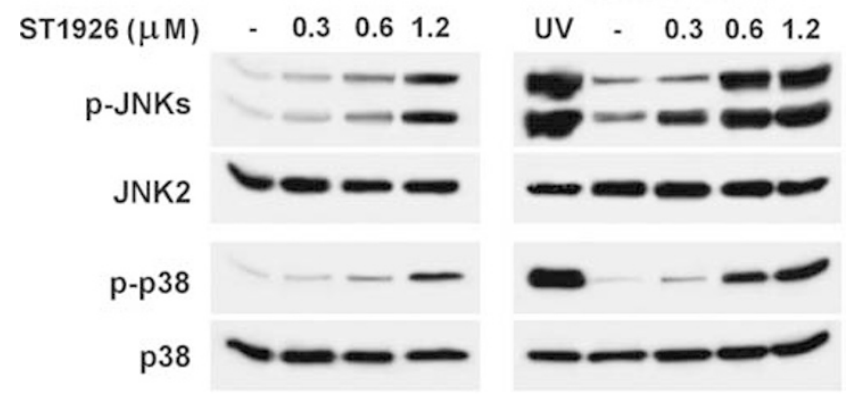

Figure 8 Modulation of MAPKs by ST1926 in IGROV-1 and IGROV-1/Pt1 cells. (a) Time course of JNK and p38 activation. (b) Dose-response effect of ST1926 on JNK and p38 activation. Cells were treated with solvent or ST1926 at equitoxic concentrations $\left({ } \mathrm{C}_{80}\right)$ for the indicated times (a) or with different concentrations of ST1926 for $6 \mathrm{~h}$ (b). UV-treated IGROV-1/Pt-1 cells represent a positive control for MAPK activation. MAPK activation and expression were analyzed in whole-cell lysates by Western blotting. Activated kinases were detected by the use of phosphospecific antibodies recognizing phospho-p44/42 ERKs ( $p$-ERK1/2), phospho-JNK (p-JNKs) and phospho p38 (p-p38). Filters were then stripped and reprobed with antibodies recognizing the respective proteins (ERK1/2, JNKs and p38)

an inhibitor able to block activation of ERK1/2, cell growth inhibition and apoptosis were enhanced in both the cell lines (Figure 9, bottom panels). These results suggest that basal activity, as in the case of ERKs, or ST1926-induced activation of MAP kinases have a protective role in IGROV-1 cells against the drug cytotoxicity. The basal activity of ERK1/2 could play a similar protective role in IGROV-1/Pt1 cells, whereas the strong activation of JNK and p38 kinases induced by ST1926 in this cell line is likely involved in other functions not restraining apoptosis.

\section{Effects on transcription factors}

Activation of MAPKs induced by ST1926 suggested that the compound could finally affect gene expression through the activation of transcription factors. Transcription factors are key mediators of a wide variety of cellular responses including pro- and antiapoptotic pathways activated in response to 


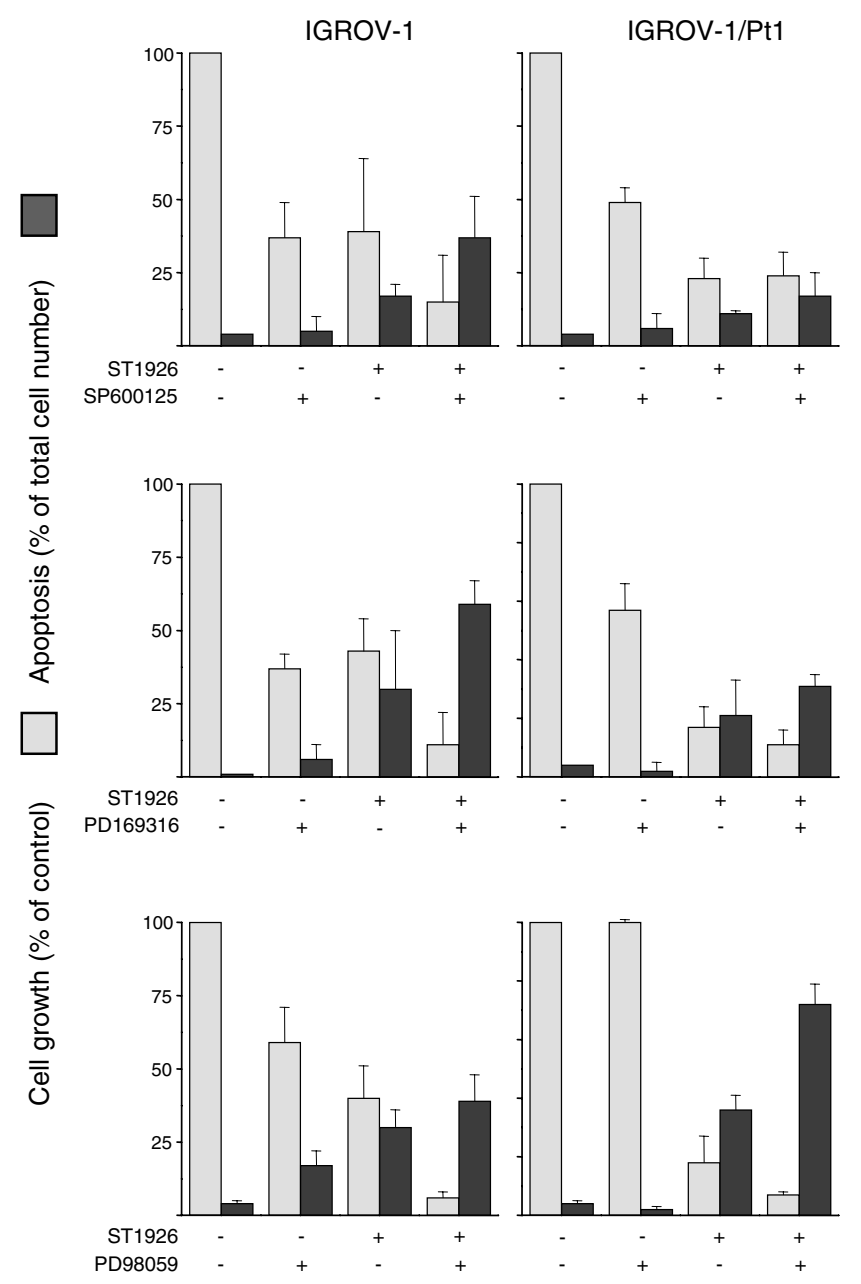

Figure 9 Effects of JNK inhibitor SP600125, $15 \mu \mathrm{M}$ (top panels), p38 inhibitor PD169316, $15 \mu \mathrm{M}$ (middle panels) and MEK inhibitor PD98059, $10 \mu \mathrm{M}$ (bottom panels), on cell growth and apoptosis in IGROV-1 and IGROV-1/Pt1 cells treated for $72 \mathrm{~h}$ with ST1926 at the $\mathrm{IC}_{50}$ and $\mathrm{IC}_{80}$, respectively (see Materials and Methods for details). Results are the mean \pm S.D. of three independent experiments. Light gray columns: cell growth (\% of control). Dark gray columns: percentage of apoptotic cells on total cell number (TUNEL assay)

cellular stress. To address this point, in adherent cells exposed to the drug $\left(\mathrm{IC}_{80}\right)$, we examined the phosphorylation of c-Jun, a major JNK substrate and component of AP-1 transcription factor complexes. ${ }^{18}$ As evidenced in Figure 10, drug treatment induced c-Jun phosphorylation at serine residues 63 and 73, a modification that is known to increase c-Jun transcription activity ${ }^{19}$ in the IGROV-1/Pt1 cell line. In these cells, drug-induced phosporylation of c-Jun, already evident following $3 \mathrm{~h}$ (not shown), produced a maximum effect at $6 \mathrm{~h}$, which remained sustained for up to $24 \mathrm{~h}$. According to drug-induced activation of JNKs, c-Jun phosphorylation was markedly increased in IGROV-1/Pt1 cells compared to IGROV-1 cells. In fact, following exposure to an equitoxic drug concentration, phospho-c-Jun was induced only transiently in IGROV-1 cells, being barely detectable only following $6 \mathrm{~h}$ of treatment.

Then, we examined the effect of ST1926 on c-Fos and ATF2 , which are partners of $c-J u n$ in the AP-1 complex. ${ }^{18}$ In the examined period of time (6-24 h), the expression of c-Fos was

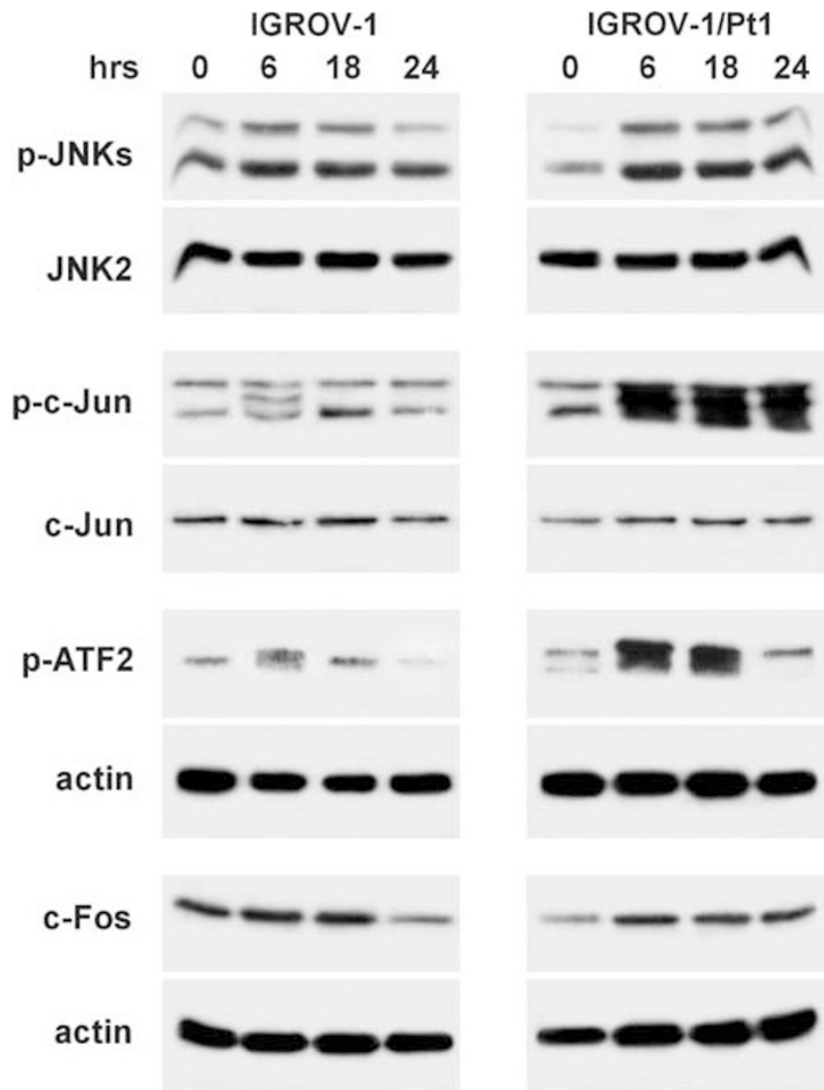

Figure 10 Effect of ST1926 on AP-1 transcription factors. The two ovarian carcinoma cell lines were treated with vehicle or ST1926 at equitoxic concentrations $\left(\mathrm{IC}_{80}\right)$ for the indicated times up to $24 \mathrm{~h}$. Cells were lysed and processed for Western blotting. Phosphospecific antibodies were used to detect the phosphorylation of JNKs, C-Jun and ATF-2 after ST1926 treatment. Protein expression was examined by the specific anti-protein antibodies. Sample loading was controlled by anti-actin blot

upregulated in ST1926-treated IGROV-1/Pt1 cells, whereas in IGROV-1 cells it was downregulated after $24 \mathrm{~h}$ of exposure to the drug. Similarly, activating phosphorylation of ATF-2 transcription factor, a well-known substrate of both JNK and p38 kinase, ${ }^{20}$ was induced in IGROV-1/Pt1 cells between 6 and $18 \mathrm{~h}$ of exposure to ST1926, whereas in the parental cell line $\mathrm{p}$-ATF-2 disappeared at $24 \mathrm{~h}$ after treatment. These results suggest that the transcriptional activity by AP-1 factors plays a relevant role in the cellular response of IGROV-1/Pt1 cells to ST1926.

\section{cDNA array analysis}

We have performed a comparative analysis of gene expression profiles in IGROV-1 cells and in the IGROV-1/Pt1 variant to investigate the modulation of genes implicated in DNA damage response and DNA repair at 4,8 or $24 \mathrm{~h}$ after addition of ST1926. Table 1 shows the selected genes, whose expression resulted at least two-fold up- or downregulated. As expected, during activation of cellular stress response, an early upregulation of c-Jun was detected in IGROV-1 cells. Upregulation of c-Jun was a more delayed event in IGROV-1/ Pt1 cells. Another key element of stress response, NF- $\kappa \mathrm{B}$, 
Table 1 Modulation of expression of genes implicated in DNA damage response

\begin{tabular}{|c|c|c|c|c|c|c|c|}
\hline \multirow[b]{2}{*}{ Gene code } & \multicolumn{3}{|c|}{ IGROV-1 } & \multicolumn{3}{|c|}{ IGROV-1/Pt1 } & \\
\hline & $4 h$ & $8 \mathrm{~h}$ & $24 \mathrm{~h}$ & $4 \mathrm{~h}$ & $8 \mathrm{~h}$ & $24 \mathrm{~h}$ & \\
\hline X42541 MC12j & $\uparrow 3.1$ & $\uparrow 2.6$ & $\uparrow 4.0$ & $\uparrow 5.1$ & & & Early growth response protein 1 (hEGR1); transcription factor ETR10 \\
\hline J04111 A01C & $\uparrow 2.5$ & $\uparrow 3.3$ & & & $\uparrow 2.7$ & $\uparrow 2.3$ & c-jun proto-oncogene; transcription factor AP-1 \\
\hline U18300 C14h & $\uparrow 2.7$ & & & & & $\uparrow 3.6$ & Damage-specific DNA-binding protein p48 subunit (DDBB P48) \\
\hline U07418 C08g & $\uparrow 5.0$ & & & $\uparrow 2.3$ & & & DNA-mismatch repair protein MLH1 \\
\hline X61498 C08j & & $\uparrow 8.0$ & & & $\uparrow 3.1$ & $\uparrow 5.8$ & NF- $\kappa$ B p100 subunit \\
\hline U35835 +CO2h & & $\uparrow 4.1$ & & & & & DNA-dependent protein kinase (DNA-PK)+DNA-PK catalytic subunit \\
\hline M60974 C09i & & $\uparrow 3.0$ & & & & & Growth arrest and DNA-damage-inducible protein (GADD45) \\
\hline M14752 Ao6e & $\downarrow 12.7$ & & & & & & c-abl, p150 \\
\hline J03250 C11e & $\downarrow 2.9$ & & & & & & DNA topoisomerase I (TOP1) \\
\hline J04088 C12e & $\downarrow 2.9$ & & & & & $\uparrow 3.4$ & DNA topoisomerase II alpha (TOP2A) \\
\hline M29039 A01d & & & $\uparrow 3.0$ & & & & jun-B \\
\hline X56681 A09c & & & $\uparrow 3.9$ & & & & jun-D \\
\hline M36067 C01g & & & & & & $\uparrow 2.9$ & DNA ligase I; polydeoxyribonucleotide synthase (ATP) \\
\hline M36089 C01h & & & & & & 2.0 & DNA-repair protein XRCC1 \\
\hline L07541 C03f & & & & & & $\uparrow 2.8$ & Replication factor C 38-kDa subunit (RFC38) \\
\hline M87339 C04f & & & & & & $\uparrow 3.0$ & Activator $137-\mathrm{kDa}$ subunit; replication factor $\mathrm{C} 37-\mathrm{kDa}$ subunit \\
\hline M30938 C14f & & & & & & $\uparrow 3.0$ & $\mathrm{Ku}$ (p70/p80) subunit; ATP-dependent DNA helicase II 86-kDa subunit \\
\hline
\end{tabular}

was upregulated in both the cell lines, but this modulation was more persistent in IGROV-1/Pt1 cells. In contrast to IGROV-1 cells, in the cisplatin-resistant subline, a number of genes involved in DNA repair were detected at $24 \mathrm{~h}$. The differential expression of DNA repair-associated genes could not be ascribed solely to a different onset of apoptosis. Indeed, at this time, most of treated cells were still adherent to the flask, and the level of apoptosis was low (30 and 10\% in IGROV-1 and IGROV-1/Pt cells, respectively). This pattern of gene expression was consistent with a more marked and persistent activation of related transcription factors in the resistant subline (Figure 10). In addition, the drug induced an early downregulation of DNA topoisomerase I and Il $\alpha$ in IGROV-1 cells, in contrast to an upregulation in IGROV-1/Pt1 cells. Again, DNA topoisomerases may have a role in DNA repair processes.

\section{Discussion}

The available evidence suggests a complex mechanism of action of atypical retinoids, including CD437 and related compounds. ${ }^{1,21-23}$ Multiple and sometimes conflicting results have been reported concerning the molecular events mediating induction of apoptosis by synthetic retinoids. ${ }^{1,3,24-26}$ Some of these agents retain a weak ability to transactivate RAR $\gamma$. However, recent studies support that CD437 does not induce apoptosis through activation of RAR $\gamma .{ }^{26}$ Such conclusion is also consistent with our recent finding that a novel related agent ST1926 is a potent inducer of apoptosis in spite of an almost complete loss of ability to transactivate RARs. ${ }^{9}$ A novel observation of our previous study was the evidence that ST1926 is able to cause DNA strand breaks after a short-term exposure $(6 \mathrm{~h})$. The precise mechanism of this type of DNA lesion remains unknown. Generation of reactive oxygen species has been reported for CD437.,24 However, in our cell system, we did not detect modifications of mitochondrial trans-membrane potential nor ROS generation by ST1926 after 3-6h exposure (data not shown). In keeping with this finding was the inability of antioxidant agents (e.g. NAC) to protect cells from ST1926-induced apoptosis (data not shown). These results do not support a DNA damage mediated by oxidative stress. DNA strand breaks were observed within a short-term exposure $(6 \mathrm{~h})$ to the drug in both IGROV-1 and IGROV-1/Pt1 cells, in spite of the different onset of apoptosis. This suggests that DNA strand breaks were not an early endonucleolytic DNA fragmentation associated with apoptosis. Recently, a report by Zhao et al. ${ }^{27}$ showed that CD437 can cause DNA adduct formation. If such adducts occur also following exposure to ST1926, DNA strand breaks found in ovarian carcinoma cells could be the consequence of the chemical DNA modification. The evidence of ST1926-induced DNA damage and the pathway of cellular response to ST1926 support that the genotoxic stress is a critical event mediating the induction of apoptosis. Several observations are consistent with this interpretation: (a) exposure of IGROV-1 cells to the drug caused activation of p53 and upregulation of p53-regulated genes (e.g., Bax); (b) IGROV-1 cells containing wild-type p53 were more susceptible to ST1926-induced apoptosis than the cisplatin-resistant subline characterized by mutant p53. As a typical DNA damage response, the activation of p53-mediated pathways could account for the increased susceptibility of IGROV-1 cells to apoptosis, induced by ST1926 as well as cisplatin; ${ }^{28}$ (c) drug-induced cell cycle perturbations are consistent with activation of DNA damage-induced checkpoints. In particular, IGROV-1 cells exhibited a more efficient G1 checkpoint, which was consistent with a functional p53, in contrast to p53 mutant IGROV-1/Pt1 cells, which showed less stringent control mechanisms at $\mathrm{G} 1$ and partially accumulated in the S phase.

JNK has been implicated in the regulation of functional activities of p53 in response to genotoxic stresses. ${ }^{29-31}$ Indeed, cellular stresses are known to cause activation of various stress-signalling pathways, including ERKs, JNKs and p38 MAPK cascades. ${ }^{31}$ In particular, JNKs phosphorylate the transcription factors of AP-1 family, including c-Jun and ATF-2, and stimulate their transcriptional activity. ${ }^{29}$ The 
activation of JNK pathway following ST1926 treatment is consistent with a cell death response to DNA damage, as observed for UV irradiation (Figure 8).

As expected, the activation of stress-activated protein kinases was also detected in IGROV-1 cells following treatment with cisplatin, a well-known genotoxic agent, thus supporting that DNA damage can activate stress-signalling pathways in our cell system (not shown). A direct comparison of cisplatin- and ST1926-induced effects is somewhat problematic, since the mechanisms involved in DNA damage recognition and signalling and stress response pathways may depend on the type and extent of genotoxic damage. ${ }^{32}$

A prolonged activation of JNK and p38 kinases has been implicated in the induction of apoptosis by other atypical retinoids. ${ }^{5}$ In the ovarian carcinoma cell system used in our study, ST1926 induced activation of both stress-activated kinases, p38 and JNK. This effect was substantially more marked in the cisplatin-resistant subline. The inhibition of p38 by PD169316 resulted in an increase of ST1926-induced apoptosis only in the parental cell line with wild-type p53. Similarly, the use of the JNK inhibitor SP600125 produced a differential effect on the two cell lines. In IGROV-1 cells, in which the inhibition of JNK pathway likely enhanced p53mediated apoptosis, ${ }^{33,34}$ the activation of the two stressrelated pathways may reflect a protective function rather than a proapoptotic role. A protective response is also a plausible explanation for the drug-induced activation of p38 and JNKs in the context characterized by 053 inactivation. Indeed, in IGROV-1/Pt1 cells, in spite of an enhanced activation of these pathways, the drug-induced apoptosis was reduced. Thus, these results could suggest an increased cell efficiency in inducing a DNA repair process. In fact, inhibition of the JNK pathway has been reported to block DNA repair and to decrease cell viability following cisplatin treatment. ${ }^{34}$ The persistent RPA-2 phosphorylation and PCNA recruitment to repair sites in treated IGROV-1/Pt1 cells, together with an early and persistent activation of AP-1 transcription factors and the upregulation of various genes implicated in DNA damage response (Table 1), are consistent with such interpretation. Thus, it appears that the influence of activation of JNK and p38 kinases on cellular response and outcome is dependent on the nature of stress and the context of various signals received by the cells. Finally, the inhibition of ERK signalling pathways resulted in an enhancement of apoptosis in both the cell lines. Taken together, these observations suggest that MAPK pathways play a protective role in response to ST1926-induced cellular stress. It is conceivable that stress response promotes DNA repair through persistent activation of AP-1, as proposed in other cell systems, ${ }^{7}$ rather than activation of survival pathways. Indeed, a lack of activation of PI3K/AKT and NF- $\kappa$ B pathways (not shown) is consistent with this interpretation.

In conclusion, the pattern of cellular response of our ovarian carcinoma cell system to the atypical retinoid ST1926 and the modulation of several genes implicated in DNA damage response support that genotoxic stress is a critical molecular event mediating induction of apoptosis by the novel agent. A p53-dependent response was clearly involved in the rapid induction of apoptosis observed in p53 wild-type cells. This pathway appeared to be regulated by stress-activated protein kinases, JNK and p38. However, the cellular fate is likely the result of contribution of multiple p53-dependent and independent pathways including an ERK-regulated signalling, as documented in both the cell lines. The unusual mechanism of action of the novel agent ST1926 and its efficacy and tolerability ${ }^{9}$ support the potential therapeutic interest for preclinical development.

\section{Materials and Methods}

\section{Drugs and antibodies}

ST1926 was prepared as described. ${ }^{9}$ SP600125 (JNK inhibitor) was purchased from Tocris (Ellisville, MO, USA), PD169316 (p38 kinase inhibitor) from Calbiochem (San Diego, CA, USA) and PD98059 (MEK-1 inhibitor) from Sigma (St. Louis, MO, USA). All the compounds were dissolved in DMSO and further diluted in culture medium (final concentration $0.5 \%$ ).

The following primary antibodies were used: anti-phospho-p38 MAP kinase (Thr 180/Tyr 182), anti-phospho-p44/42 MAP kinase (Thr 202/Tyr 204) and anti-phospho-c-Jun (Ser 63) (New England BioLabs, Beverly, MA, USA); anti-phospho-JNK (Thr 183/Tyr 185), anti-Bcl-2, anti-Shc (C20) and anti-p38 (C-20) (Santa Cruz Biothechnology, Santa Cruz, CA, USA); anti-c-Fos anti-MAP kinase 1/2 (ERK 1/2-CT) (Upstate Biotechnology, Lake Placid, NY, USA); anti-phospho ATF2 (Thr 71) and anti-SAPK/ JNK (Cell Signaling Technology, Beverly, MA, USA); anti-p53 and anti-Bcl2 (Dako, Glostrup, Denmark); anti-Bax, anti-caspase 3 and anti-caspase 8 (Pharmigen, Becton Dickinson, Mountain View, CA, USA); anti-caspase 9, anti-RPA-2, anti-PCNA and anti-p21 (Neomarker, Union city, CA, USA); anti-PARP (Oncogene Science, Uniondale, NY, USA); anti-actin (Sigma).

\section{Cell lines and culture conditions}

The human ovarian carcinoma cell line IGROV-1 and the cisplatinresistant subline IGROV-1/Pt1, selected by exposure to increasing cisplatin concentrations, ${ }^{28,35}$ were maintained in RPMl-1640 (BioWhittaker, Verviers, Belgium) supplemented with 10\% FCS (LifeTechnologies, Inc., Gaithersburg, MD, USA). The cisplatin resistance index (ratio between the $\mathrm{IC}_{50}$ of the resistant and sensitive cell lines) was 14. The cisplatin-resistant subline was characterized by the simultaneous presence of two mis-sense mutations on different alleles found at codons 270 and 282. Since the involved residues are in the DNA-binding domain, the mutations result in p53 inactivation. ${ }^{28,35}$

\section{Antiproliferative activity}

Cell sensitivity to ST1926 was determined by cell growth-inhibition assay: IGROV-1 and IGROV-1/Pt1 were seeded into six-well plates in duplicate and, $24 \mathrm{~h}$ later, exposed to the drug for $72 \mathrm{~h}$. Then, trypsinized cells were counted by a Coulter counter (Coulter Electronics, Luton, UK). The $\mathrm{IC}_{50}$ values $( \pm$ S.D.) were calculated from the dose-response curves.

\section{Cell cycle distribution and apoptosis}

Cells were exposed, $24 \mathrm{~h}$ after seeding, to different drug concentrations. Adherent and floating cells were collected at different time points, fixed in $70 \%$ ethanol and stained with $10 \mu \mathrm{g} / \mathrm{ml}$ propidium iodide (PI) (Sigma) in PBS containing RNAse (66 U/ml) (Sigma). The cell cycle distribution was analyzed on PI stained cells by FACScan flow cytometer. Apoptosis was evaluated by TUNEL assay. Floating and adherent cells were fixed in 
paraformaldehyde, permeabilized in a solution of $0.1 \%$ Triton X-100 in $0.1 \%$ sodium citrate, and then incubated in the TUNEL reaction mix (Boehringer Mannheim Ingelheim, Germany) for $1 \mathrm{~h}$. After washing, the samples were analyzed by FACScan flow cytometer equipped with an argon laser. The percentages of apoptosis and of cell cycle distribution were calculated on at least 10000 cells, and analyzed by Lysis II software (Becton Dickinson).

\section{Alkaline elution assay}

Cells were labelled with $0.08 \mu \mathrm{Ci} / \mathrm{ml}$ of ${ }^{14} \mathrm{C}$-thymidine (Amersham Bioscences, Amersham, UK) at $37^{\circ} \mathrm{C}$ for $30 \mathrm{~h}$, and then incubated for $18 \mathrm{~h}$ in the absence of labelled thymidine to chase the DNA-incorporated radioactivity. Cells were then treated for $6 \mathrm{~h}$ with ST1926, lysed in the presence of proteinase $\mathrm{K}(0.5 \mathrm{mg} / \mathrm{ml})$ and harvested to perform the alkaline elution assay. ${ }^{36}$ As reference, one sample of cells was irradiated with a ${ }^{137} \mathrm{Cs}$ source $(1000 \mathrm{rad})$ to produce a known frequency of $\gamma$-rayinduced DNA single-strand breaks.

\section{Immunocytometric determination of PCNA recruitment}

After 6 and $24 \mathrm{~h}$ of treatment with ST1926, control and treated cells (around $1 \times 10^{6}$ cells/sample) were harvested and lysed in cold hypotonic solution to release soluble PCNA, as described. ${ }^{37}$ Cells were incubated with anti-PCNA antibody and stained with fluorescein isothiocyanate (FITC)-conjugated anti-mouse IgG (Sigma). For DNA counterstaining, cells were incubated in PBS containing $5 \mu \mathrm{g} / \mathrm{ml} \mathrm{PI}$ and $1 \mathrm{mg} / \mathrm{ml}$ RNase A (Sigma). In all, $10^{4}$ cells were analyzed for each sample by FACScan flow cytometer (Becton Dickinson).

\section{Western blot analysis}

If not otherwise indicated, cells were treated for the indicated times with solvent (DMSO) or with ST1926 at the concentrations corresponding to the $\mathrm{IC}_{80}$ (determined after $72 \mathrm{~h}$ exposure). In short-term experiments $(<24 \mathrm{~h})$, analysis was performed in adherent cells, since the number of floating cells were negligible. After long-term exposure $(\geqslant 48 \mathrm{~h}$ ), floating and adherent cells were collected. Cells were rinsed twice with cold PBS added with sodium orthovanadate $0.1 \mathrm{mM}$ and lysed in hot sodium dodecyl sulfate (SDS) sample buffer $(0.125 \mathrm{M}$ Tris- $\mathrm{HCl}$ pH 6.8, 5\% SDS) containing $1 \mathrm{mM} \mathrm{PMSF}, 10 \mu \mathrm{g} / \mathrm{ml}$ pepstatin, $12.5 \mu \mathrm{g} / \mathrm{ml}$ leupeptin, $100 \mathrm{KIU}$ aprotinin, $1 \mathrm{mM}$ sodium orthovanadate and $1 \mathrm{mM}$ sodium molybdate. Cell lysates were boiled for $2 \mathrm{~min}$ and sonicated. An aliquot was taken to determine the protein concentration (BCA assay-Pierce, Rockford, IL, USA). Samples were then adjusted for the Laemmli composition ${ }^{38}$ and further boiled for $2 \mathrm{~min}$. Whole-cell extracts were separated on SDS-PAGE and then transferred to nitrocellulose filters. After staining with Ponceau $S$ (Sigma) to control protein loading and transfer, filters were incubated with primary antibodies at $4^{\circ} \mathrm{C}$ overnight. Immunoreactive bands were revealed by horseradish peroxidase-conjugated secondary antibodies using enhanced chemiluminescence detection systems (Amersham) or Pierce (Rockford, IL, USA). When the drug treatment was combined with MAPK inhibitors, cells were preincubated with 10-15 $\mu \mathrm{M} \mathrm{SP} 600125$ for $15 \mathrm{~min}$, or with 10-15 $\mu \mathrm{M}$ PD169316 for 90 min or with $10 \mu \mathrm{M}$ PD98059 for $30 \mathrm{~min}$. In parallel experiments, the efficacy of inhibition of MAPK pathways was assessed biochemically by the following readings: inhibition of basal and drug-induced c-Jun phosphorylation by SP600125, inhibition of ERK1/2 phosphorylation by PD98059, or inhibition of p38 phosphorylation ${ }^{5}$ in cells treated with PD169316.

\section{CDNA array hybridization}

Total RNA was extracted from adherent cells using Trizol reagent (Life Technologies, Rockville, MD, USA) according to the manufacturer's instructions. RNA was treated with DNase (Ambion Inc.), and was checked for quality by electrophoresis on denaturing formaldehyde/agarose gel, as well as by $A_{260} / A_{280}$ ratios. Poly A + RNA enrichment, ${ }^{32} \mathrm{P}$-labelled cDNA probe preparation and filter hybridization were carried out as recommended by Clontech. Nylon membranes (Atlas Human Array 1.2 Clontech) consist of 1176 human cDNA fragments, organized into broad functional groups. A complete list of the gene included on the membranes is available on the Clontech Web site (http://www.clontech.com). The results of array hybridization were evaluated using phosphorimager (Molecular Dynamics, Sunnyvale, CA, USA) and analyzed with AtlasImage 2.0 software package (Clontech).

\section{Acknowledgements}

This work was partially supported by the Associazione Italiana Ricerca sul Cancro, Milan, the Ministero della Salute, Rome, the Consiglio Nazionale delle Ricerche, Rome and the Fondazione Thomas Hoepli.

\section{References}

1. Fontana JA and Rishi AK (2002) Classical and novel retinoids: their targets in cancer therapy. Leukemia 16: 463-472

2. Hsu CA, Rishi AK, Xiao S-L, Gerald TM, Dawson MI, Schiffer C, Reichert U, Shroot B, Poirer GC and Fontana JA (1997) Retinoid induced apoptosis in leukemia cells through a retinoic acid nuclear receptor-independent pathway. Blood 89: 4470-4479

3. Sun S-Y, Yue P, Chandraratna RAS, Tesfaigzi Y, Hong WK and Lotan R (2000) Dual mechanisms of action of the retinoid CD437: nuclear retinoic acid receptor-mediated suppression of squamous differentiation and receptorindependent induction of apoptosis in UMSCC22B human head and neck squamous cell carcinoma cells. Mol. Pharmacol. 58: 508-514

4. Zang Y, Beard RL, Chandraratna RAS and Kang JX (2001) Evidence of a lysosomal pathway for apoptosis induced by the synthetic retinoid CD437 in human leukemia HL-60 cells. Cell Death Differ. 8: 477-485

5. Ortiz MA, Lopez-Hernandez FJ, Bayon Y, Pfhal M and Piedrafita FJ (2001) Retinoid-related molecules induce cytochrome $c$ release and apoptosis through activation of c-Jun $\mathrm{NH}_{2}$-terminal kinase/p38 mitogen-activated protein kinases. Cancer Res. 61: 8504-8512

6. Sun S-Y, Yue P, Chen X, Hong WK and Lotan R (2002) The synthetic retinoid CD437 selectively induces apoptosis in human lung cancer cells while sparing normal human lung epithelial cells. Cancer Res. 62: 2430-2436

7. Gjerset RA, Lebedeva S, Haghighi A, Turla ST and Mercola D (1999) Inhibition of the Jun kinase pathway blocks DNA repair, enhances p53-mediated apoptosis and promotes gene amplification. Cell Growth Differ. 10: 545-554

8. Levresse V, Marek L, Blumberg D and Heasley LE (2002) Regulation of platinum-compound cytotoxicity by the c-Jun N-terminal kinase and c-Jun signaling pathway in small-cell lung cancer cells. Mol. Pharmacol. 62: 689-697

9. Cincinelli R, Dallavalle S, Merlini L, Penco S, Pisano C, Carminati P, Giannini G, Vesci L, Gaetano C, Illy B, Zuco V, Supino R and Zunino F (2003) A novel atypical retinoid endowed with proapoptotic and antitumor activity. J. Med. Chem. 46: 909-912

10. Fadeel B, Orrenius $S$ and Zhivotovsky B (2000) The most unkindest cut of all: on the multiple roles of mammalian caspases. Leukemia 14: 1514-1525

11. Li XS, Rishi AK, Shao ZM, Dawson MI, Jong L, Shroot B, Reichert U, Ordonez J and Fontana JA (1996) Posttranscriptional regulation of p21WAF1/CIP1 expression in human breast carcinoma cells. Cancer Res. 56: 5055-5062 
12. Amundson SA, Myers TG and Fornace AJ (1998) Roles of $p 53$ in growth arrest and apoptosis: putting on the brakes after genotoxic stress. Oncogene 17 3287-3299

13. Wang H, Guan J, Wang H, Perrault AR, Wang $Y$ and lliakis G (2001) Replication protein A2 phosphorylation after DNA damage by the coordinated action of ataxia telangiectasia-mutated and DNA-dependent protein kinase. Cancer Res. 61: 8554-8563

14. Stivala LA, Riva F, Cazzalini O, Savio M and Prosperi E (2001) p21waf1/ cip1-null human fibroblasts are deficient in nucleotide excision repair downstream the recruitment of PCNA to DNA repair sites. Oncogene 20: 563-570

15. Chen Y-R, Wang X, Templeton D, Davis RJ and Tan T-H (1996) The role of CJun N-terminal kinase (JNK) in apoptosis induced by ultraviolet $\mathrm{C}$ and $\gamma$ radiation. J. Biol. Chem. 271: 31929-31936

16. Bennett BL, Sasaki DT, Murray BW, O'Leary EC, Sakata ST, Xu W, Leisten JC, Motiwala A, Pierce S, Satoh Y, Bhagwat SS, Manning AM and Anderson DW (2001) SP600125, an anthrapyrazolone inhibitor of Jun N-terminal kinase. Proc. Natl. Acad. Sci. USA 98: 13681-13686

17. Kamakura S, Moriguchi T and Nishida E (1999) Activation of the protein kinase ERK5/BMK1 by receptor tyrosine kinases. J. Biol. Chem. 274: 26563-26571

18. Shaulian $E$ and Kerin M (2001) AP-1 in cell proliferation and survival. Oncogene 20: 2390-2400

19. Mechta-Grigoriou F, Gerald D and Yaniv M (2001) The mammalian Jun proteins: redundancy and specificity. Oncogene 20: 2378-2389

20. Davis RJ (2000) Signal transduction by the JNK group of MAP kinases. Cell 103: $239-252$

21. Zhao $X$ and Spanjaard RA (2003) The proapoptotic action of the retinoid CD437/AHPN: diverse effects, common basis. J. Biomed. Sci. 10: 44-49

22. Holmes WF, Soprano DR and Soprano KJ (2002) Elucidation of molecular events mediating induction of apoptosis by synthetic retinoids using a CD437resistant ovarian carcinoma cell line. J. Biol. Chem. 277: 45408-45419

23. Altucci $L$ and Gronemeyer $H$ (2001) The promise of retinoids to fight against cancer. Nat. Rev. 1: 181-193

24. Marchetti P, Zamzami N, Joseph B, Schraen-Maschke S, Mèreau-Richard C Costantini P, Mètivier D, Susin SA, Kroemer G and Formstecher P (1999) The novel retinoid 6-[3-(1-adamantyl)-4-hydroxyphenyl]-2-naphtalene carboxylic acid can trigger apoptosis through a mitochondrial pathway independent of the nucleus. Cancer Res. 59: 6257-6266

25. Li Y, Lin B, Agadir A, Liu R, Dawson Ml, Reed JC, Fontana JA, Bost F, Hobbs PD, Zheng Y, Chen G-Q, Shroot B, Mercola D and Zhang X-K (1998) Molecular determinants of AHPN (CD437)-induced growth arrest and apoptosis in human lung cancer cell lines. Mol. Cell Biol. 18: 4719-4731
26. Zhang Y, Dawson MI, Mohammad R, Rishi AK, Farhana L, Feng K-C, Leid M, Peterson V, Zhang X-K, Edelstein M, Eilander D, Biggar S, Wall N, Reichert U and Fontana JA (2002) Induction of apoptosis of human B-CLL and ALL cells by a novel retinoid and its nonretinoidal analog. Blood 100: 2917-2925

27. Zhao X, Demary K, Wong L, Vaziri C, McKenzie AB, Eberlein TJ and Spanjaard RA (2001) Retinoic acid receptor-independent mechanism of apoptosis of melanoma cells by the retinoid CD437 (AHPN). Cell Death Differ. 8: 878-886

28. Perego P, Giarola M, Righetti SC, Supino R, Caserini C, Delia D, Pierotti MA, Miyashita T, Reed JC and Zunino F (1996) Association between cisplatin resistance and mutation of p53 gene and reduced bax expression in ovarian carcinoma cell systems. Cancer Res. 56: 556-562

29. Herr I and Debatin K-M (2001) Cellular stress response and apoptosis in cancer therapy. Blood 98: 2603-2614

30. Buschmann T, Potapova O, Bar-Shira A, Ivanov VN, Fuchs SY, Henderson S, Fried VA, Minamoto T, Alarcon-Vargas D, Pincus MR, Gaarde WA, Holbrook $\mathrm{NJ}$, Shiloh $\mathrm{Y}$ and Ronai Z (2001) Jun $\mathrm{NH}_{2}$-terminal kinase phosphorylation of p53 on Thr-81 is important for p53 stabilization and transcriptional activities in response to stress. Mol. Cell Biol. 21: 2743-2754

31. Martindale $\mathrm{JL}$ and Holbrook NJ (2002) Cellular response to oxidative stress: signalling for suicide and survival. J. Cell Physiol. 192: 1-15

32. Gatti L, Supino R, Perego P, Pavesi R, Caserini C, Carenini N, Righetti SC, Zuco V and Zunino $F(2002)$ Apoptosis and growth arrest induced by platinum compounds in U2-OS cells reflect a specific DNA damage recognition associated with a different p53-mediated response. Cell Death Differ. 9: 13521359

33. Levresse V, Marek L, Blumberg D and Heasley LE (2002) Regulation of platinum-compound cytotoxicity by the c-Jun N-terminal kinase and c-Jun signalling pathway in small-cell lung cancer cells. Mol. Pharmacol. 62: 689-697

34. Gjerset RA, Lebedeva S, Haghighi A, Turla ST and Mercola D (1999) Inhibition of the Jun kinase pathway blocks DNA repair, enhances p53-mediated apoptosis and promotes gene amplification. Cell Growth Differ. 10: 545-554

35. Perego P, Romanelli S, Carenini N, Magnani I, Leone R, Bonetti A, Paolicchi A and Zunino $F$ (1998) Ovarian cancer cisplatin-resistant cell lines: multiple changes including collateral sensitivity to taxol. Ann. Oncol. 9: 423-430

36. Kohn KW, Ewig RAG, Erickson LC and Zwelling LA (1981) DNA repair. In A Laboratory Manual of Research Procedures Friedberg, EC and Hanawalt PC (eds), Vol. 1 (Part B) (New York: Marcel-Dekker), pp. 370-401

37. Frouin I, Prosperi E, Denegri M, Negri C, Donzelli M, Rossi L, Riva F, Stefanini $M$ and Scovassi Al (2001) Different effects of methotrexate on DNA mismatch repair proficient and deficient cells. Eur. J. Cancer 37: 1173-1180

38. Laemmli UK (1970) Cleavage of structural proteins during the assembly of the head of bacteriophage T4. Nature 227: 680-685 